\title{
Public awareness level and occurrence of pharmaceutical residues in drinking water with potential health risk: a study from Kajang (Malaysia)
}

\begin{abstract}
Studies on the occurrence of pharmaceutical residues in drinking water were conducted especially in developed countries. However, limited studies reported the occurrence of pharmaceutical residues in developing countries. Thus, this study is conducted to fill the knowledge gap of pharmaceutical residue occurrences in developing countries, particularly in Malaysia, along with public awareness level and its potential human health risk. This study investigates public awareness level of drinking water quality and pharmaceutical handling, the occurrence of nine pharmaceutical residues (amoxicillin, caffeine, chloramphenicol, ciprofloxacin, dexamethasone, diclofenac, nitrofurazone, sulfamethoxazole, and triclosan) and potential human health risks in drinking water from Kajang (Malaysia) using commercially competitive enzyme-linked immunosorbent assay kits. In general, the public awareness level of Kajang population showed poor knowledge $(82.02 \%)$, and less positive attitude $(98.88 \%)$ with a good practice score $(57.3 \%)$. Ciprofloxacin was detected at the highest concentration $(0.667$ $\mathrm{ng} / \mathrm{L})$ while amoxicillin was at the lowest concentration $(0.001 \mathrm{ng} / \mathrm{L})$ in drinking water from Kajang (Malaysia). Nevertheless, all the reported occurrences were lower than previous studies conducted elsewhere. There was no appreciable potential human health risk for all the pharmaceutical residues as the risk quotient (RQ) values were less than 1 (RQ $<1)$. The results of this study will provide authorities with quantitative knowledge and resources to improve drinking water risk management and regulation in Malaysia.
\end{abstract}

Keyword : Drinking water; Health risks; Pharmaceutical residues; Public awareness. 